\title{
Silver Nanoparticles under Nanosecond Pulsed Laser Excitation as an Intensity Sensitive Saturable Absorption to Reverse Saturable Absorption Switching Material
}

\author{
Edappadikkunnummal Shiju ${ }^{1}$, Kaniyarakkal Sharafudeen ${ }^{2, *}$, T. M. Remya ${ }^{3}$, N. K. Siji Narendran ${ }^{4}$, \\ Palengara Sudheesh ${ }^{5}$ and Vijayakumar Sadasivan Nair ${ }^{6}$
}

1 International School of Photonics, Cochin University of Science and Technology, Kochi 682022, Kerala, India; shijue@cusat.ac.in

2 Department of Physics, Kuwait College of Science and Technology, Doha Area, Kuwait City 27235, Kuwait

3 Department of Applied Chemistry, Cochin University of Science and Technology, Kochi 682022, Kerala, India; remyamurali@cusat.ac.in

4 Department of Physics, T K M M College, Nangyarkulangara 690513, Kerala, India; sijinarendran@gmail.com

5 Department of Physics, N S S College, Manjeri 676122, Kerala, India; sudheeshp9@gmail.com

6 PG and Research Department of Physics, N S S College, Pandalam 689501, Kerala, India; jevijay@gmail.com

* Correspondence: s.valappil@kcst.edu.kw or sharafkn@gmail.com

Citation: Shiju, E.; Sharafudeen, K.; Remya, T.M.; Siji Narendran, N.K.; Sudheesh, P.; Sadasivan Nair, V. Silver Nanoparticles under Nanosecond Pulsed Laser Excitation as an Intensity Sensitive Saturable Absorption to Reverse Saturable Absorption Switching Material. Photonics 2021, 8, 413. https://doi. org $/ 10.3390 /$ photonics 8100413

Received: 30 August 2021 Accepted: 22 September 2021 Published: 28 September 2021

Publisher's Note: MDPI stays neutral with regard to jurisdictional claims in published maps and institutional affiliations.

Copyright: () 2021 by the authors. Licensee MDPI, Basel, Switzerland. This article is an open access article distributed under the terms and conditions of the Creative Commons Attribution (CC BY) license (https:// creativecommons.org/licenses/by/ $4.0 /)$.

\begin{abstract}
Optical nonlinearity involved switching draws an important consideration in nonlinear optical studies. Based on that, we explored nonlinear absorption processes in silver nanoparticles synthesized by liquid phase laser ablation technique employing a second harmonic wavelength $(532 \mathrm{~nm})$ of Q switched Nd:YAG laser pulses with $7 \mathrm{~ns}$ pulse width and $10 \mathrm{~Hz}$ repetition rates. The typical surface plasmon resonance induced absorption $(\sim 418 \mathrm{~nm})$ confirmed the formation of $\mathrm{Ag}$ NPs. The Z-scan technique was used to study the nonlinear optical processes, employing the same laser system used for ablation. Our study reveals that there is an occurrence of a saturable to reverse saturable absorption switching activity in the Ag nanoparticles, which is strongly on-axis input intensity dependent as well. The closed aperture Z-scan analysis revealed the self-defocusing nature of the sample.
\end{abstract}

Keywords: pulsed laser ablation; Z-scan; nonlinear absorption; nonlinear refraction; optical limiting

\section{Introduction}

Metal nanoparticles (NPs) or nanostructured materials are drawing major attention in the research field as well as in industry due to their unique and better properties compared to their bulk counterparts [1-4]. This is mainly due to the surface plasmon resonance (SPR) which is the collective oscillation of electron cloud in the conduction band in conformity with the quantum effects originated from the reduced dimensionality. When optically induced, the SPR of nanoparticles enhances the local field intensity, which in turn enhances the intense light matter interaction. There are many studies available on the interaction between metal NPs and intense laser beam which show that different nonlinear mechanisms, like two photon absorption (TPA), saturable absorption (SA), excited state absorption, and optical Kerr nonlinearities, are accountable for the NLA property [5-7]. In a nonlinear optical material, when the absorptive property diminishes with an increase in input intensity caused by the depletion of ground states, it is termed as SA. If a nonlinear absorbing material's excited state absorption is larger than the ground state absorption, the absorptive property enhances with an increase in input intensity, and the material is said to exhibit reverse saturable absorption (RSA). The SA mechanism plays a significant role in the observed nonlinearity at sufficiently high input fluence of the interacting laser pulse; once the input fluence crosses the threshold value, the reverse saturable absorption (RSA) overtakes the SA process. The RSA process occurs because of the strong absorption 
occurring in the nonlinear medium at higher input fluence, via excited state absorption. There is a paucity of reports available on the competitive behavior of SA and RSA [8].

In the field of fundamental and technological research, nano-materials with excellent third order optical nonlinearity have a substantial role [8-11]. The linear as well as nonlinear optical (NLO) responses of noble NPs strongly depend on SPR. The major aspect in the linear optical response of these systems is the excitation of the SPR, which stimulates the absorption band whose spectral amplitude, location, and band-width depend on the NPs shape, size, and concentration in the medium [7]. Several factors such as enhanced surface area, the size, shape, larger refractive index changes in dielectric medium surrounding the NPs, and laser intensity are responsible for signal enhancement in the NLO investigations $[4,7,12]$. The metal NPs exhibit fast response times and are either saturable absorption (SA) based or reverse saturable absorption (RSA) based [10,11]. Additionally, these NPs can be easily synthesized and have attractive thermal and chemical stability [7]. The improved NLO activities of the NPs due to their SPR and quantum size effects can have a number of applications like, optical switching, SERS, data storage, and optical limiting. [1,4,6,11-14]. Compared to the other noble metal NPs, the Ag NPs are attractive systems since the intrinsic loss of plasmonic energy of Ag NPs at the visible region of the spectrum is comparatively low [6].

There are number of methods available to synthesize the metal NPs, with the frequently used techniques being laser ablation, chemical synthesis, ion implantation, and sol-gel methods [6,7]. The present work employed the pulsed laser ablation (PLA) method to synthesize the Ag NPs. PLA is a clean physical method whereby NPs are formed by ablating individual targets kept either in a gaseous or liquid atmosphere by a strong laser beam $[4,11,12,15,16]$. PLA has a number of advantages over other NPs synthesis methods, in that it is a chemically clean, quick, and straight forward method. It does not require separate setups to generate extreme conditions like high pressure and temperature. Moreover, it does not involve long reaction times or a multistep synthesis process. The morphology (size and shape) of the synthesized NPs strongly depends on the wavelength, energy, repetition rate, and pulse width of the interacting laser as well as the nearby dielectric medium [16].

Here, we report a nonlinear optical analysis of Ag NPs using the Z-scan technique. The studied Ag NPs were synthesized by the PLA technique. The source of excitation for PLA and Z-scan analysis was a Q switched Nd: YAG laser working at $532 \mathrm{~nm}$. The Z-scan results of the Ag NPs, revealed a switching of NLA behavior from SA to RSA as we increased the intensity/fluence of the laser beam. The reason behind the switching could be the interplay between ground state SPR band bleach and excited state absorption. The negative nonlinearity (peak followed by valley) of the synthesized Ag NPs is revealed from the closed aperture Z-scan signature. The intensity reliant NLA switching actions of Ag NPs can probably make use of a series of photonic applications; optical switching, pulse compression, mode locking, and optical limiter are a few among them [17,18]. This work reports that with the excitation of ns laser pulses at $532 \mathrm{~nm}$ wavelength in Ag NPs colloids, is was a switching behavior from SA to RSA when the input intensity is at $0.207 \mathrm{GW} / \mathrm{cm}^{2}$. In comparison to the previously reported value of $0.04 \mathrm{GW} / \mathrm{cm}^{2}$ [19], the intensity threshold observed is relatively large and it ensures that the SA behavior can be observed over a large range of intensity inputs [18-20].

\section{Experimental Method}

Silver nanoparticles (AgNPs) were synthesized in the dimethyl formamide solution by the laser ablation technique; the schematic is shown in the Figure 1a. A solid silver target of $1 \mathrm{~mm}$ thickness (>99\%) was kept at the bottom of a glass beaker filled with $10 \mathrm{~mL}$ DMF. A frequency doubled laser (Nd: YAG) producing $532 \mathrm{~nm}$ laser pulses of $7 \mathrm{~ns}$ pulse width and $10 \mathrm{~Hz}$ repetition rate was used as the source of light in the experiment. The laser beam is focused on the target with a $10 \mathrm{~cm}$ focal length lens. The ablation experiment was performed with incident energy of $20 \mathrm{~mJ}$ for $20 \mathrm{~min}$. 


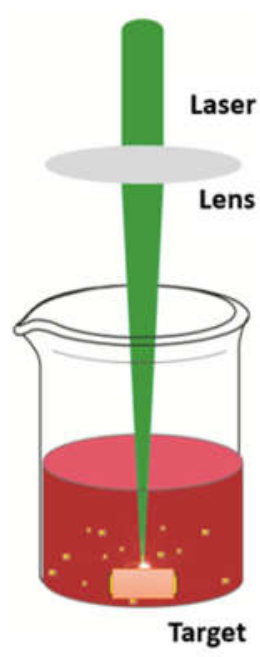

(a)

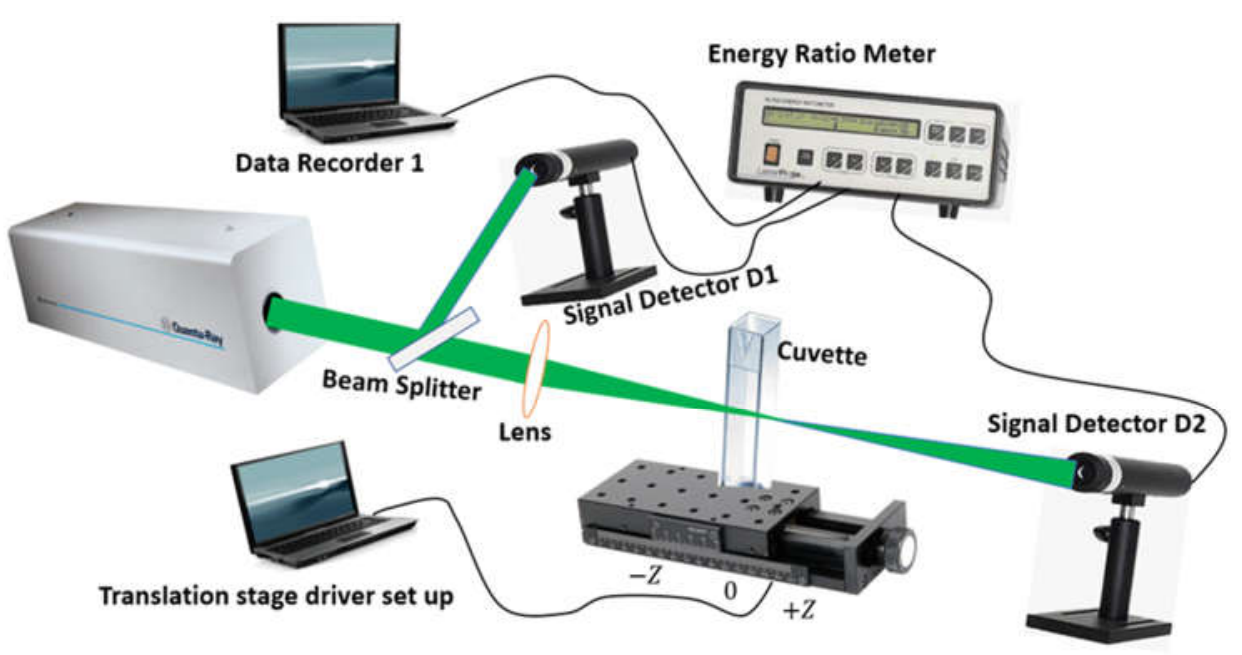

(b)

Figure 1. Schematic of (a) Laser ablation setup and (b) Z-scan experimental arrangements.

For nonlinear optical studies, a Q switched Nd:YAG laser (Quanta-Ray INDI-40) delivering pulses of $7 \mathrm{~ns}$ pulse width and $10 \mathrm{~Hz}$ at $532 \mathrm{~nm}$ wavelength was focused with a convex lens of $15 \mathrm{~cm}$ focal length. The Z-scan experimental arrangement is depicted in Figure 1b. The sample was placed in a quartz cuvette with an optical path length of $1 \mathrm{~mm}$ and mounted on a computer supported translational stage, to ensure the accurate movement of the sample over a distance of $20 \mathrm{~mm}$ on either side of the focus. The laser beam was divided into two using a beam splitter; one was used as reference beam and the other was guided to the sample. Pyroelectric detectors (RjP-735, Laser Probe. Corp., Utica, NY 13501, USA) were used to measure the reference and transmitted beam energies and both the collected beams were sent to an energy ratio meter (Rj-7620, Laser Probe Corp., Utica, NY 13501, USA). With the open aperture Z-scan technique, the transmitted beam of light was fully collected by keeping the detector aperture free. With the closed aperture Z-scan technique, an aperture was employed in front of the detector so that the changes in optical transmission could be measured as a result of the combined nonlinear absorption and nonlinear refractive index induced phase change. The normalized transmittance in the closed aperture experiment was recorded at different sample positions and we observed a pre-focal peak followed by a post focal valley. We then applied the division method on the normalized closed and open aperture curve to obtain pure refractive traces. Cumulative thermal contributions were minimized by conducting the experiment in a single shot mode.

\section{Results and Discussion}

Using a spectrophotometer (Shimadzu-UV 2450), the absorption spectrum of the prepared Ag NPs was recorded and is shown in Figure 2a. The location of Ag NPs SPR band was found to be around $418 \mathrm{~nm}$ and the occurrence of this peak was due to the size of the Ag NPs. Figure $2 b$ shows the SEM image of the prepared Ag NPs and the spherical morphology of the NPs are revealed from the image. 

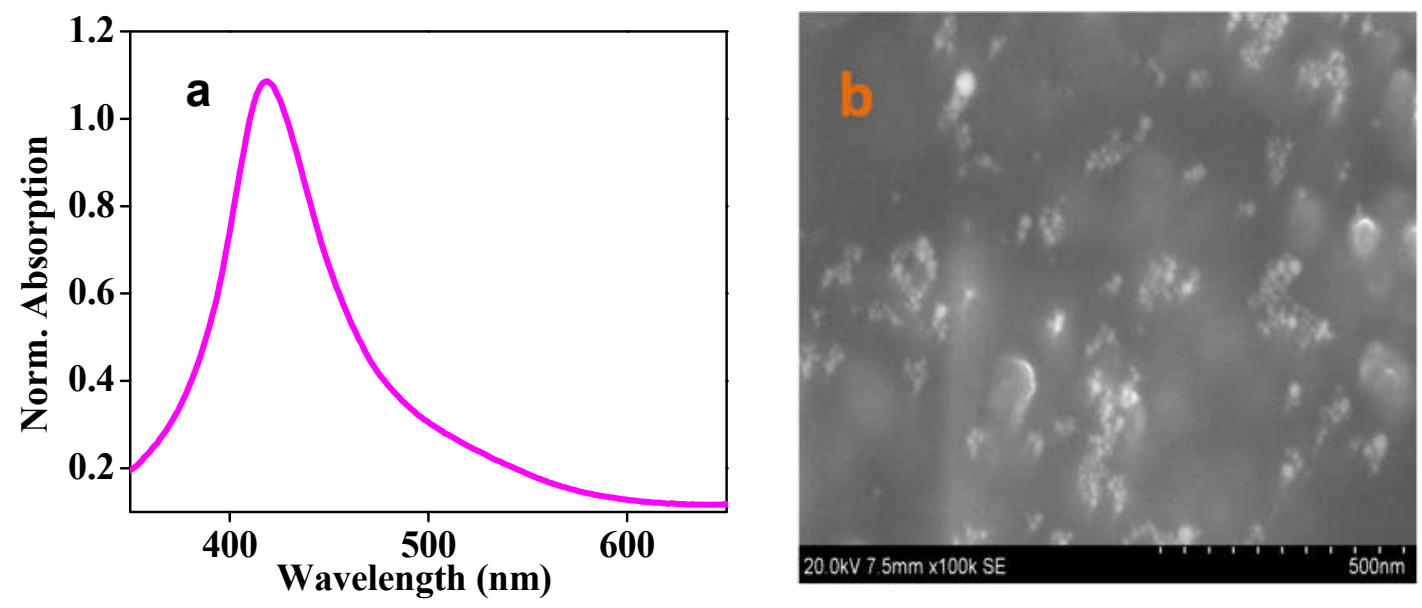

Figure 2. (a) Linear absorption and (b) SEM image of prepared Ag NPs.

The morphology of the synthesized Ag NPs was characterized using transmission electron microscopy (TEM) (JEOL, JEM-2100). TEM sample preparation was done by dropping the Ag NPs solution on top of a carbon cotted copper grid band and drying it out before the examination. Figure 3a displays the TEM image of the NPs; the formation of sphere-shaped Ag NPs are evident from the obtained TEM image. The electron diffraction (SAED) analysis of Ag conducted on a selected area is given in Figure 3b. The d values estimated from the SAED data match well with the literature values of (111), (200), and (220) plans of the fcc Ag crystal [12].
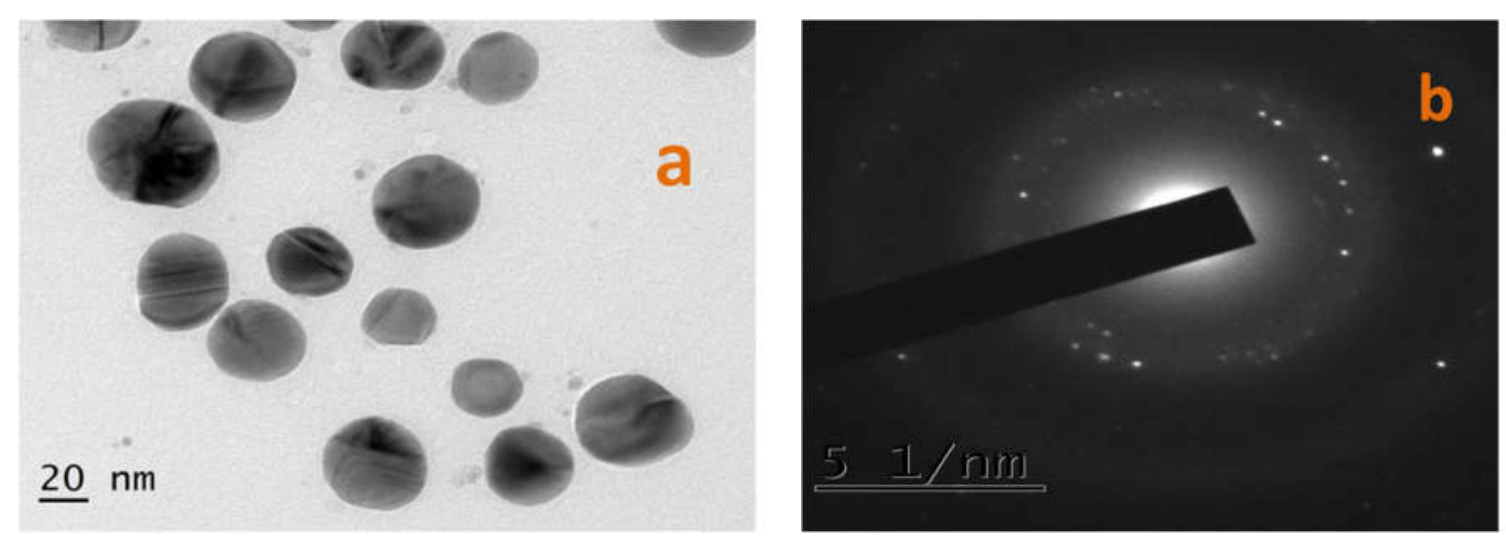

Figure 3. (a) TEM image and (b) SAED pattern of Ag nanoparticles.

There are many studies on the influence of SPR bands of Ag NPs on its NLO properties [18-20]. The size, shape, and dielectric value of the nearby medium are factors that affect the position and width of SPR bands $[1,4,7]$. The open aperture Z-scan signatures of Ag NPs (Linear transmittance $\sim 80 \%$ ) at various on axis input intensities are given in Figure $4 a, b$. The transmittance variation for Gaussian pulse propagation in a nonlinear optical medium based on Equation (1) was employed [4,7,17], and then the experimental data were fitted based on Equation (1):

$$
\frac{d I}{d z}=-\left(\frac{\alpha_{0}}{1+\frac{I}{I_{s}}}\right) I-\beta_{e f f} I^{2}
$$

where $I$ and $I_{S}$ are input and saturation intensities, $\alpha_{0}$ and $\beta_{\text {eff }}$ are linear and nonlinear absorption coefficients, and $\mathrm{z}^{\prime}$ is the propagation distance inside the sample, respectively. Here, the first term accounts for SA effects and the second term accounts for the RSA process. 

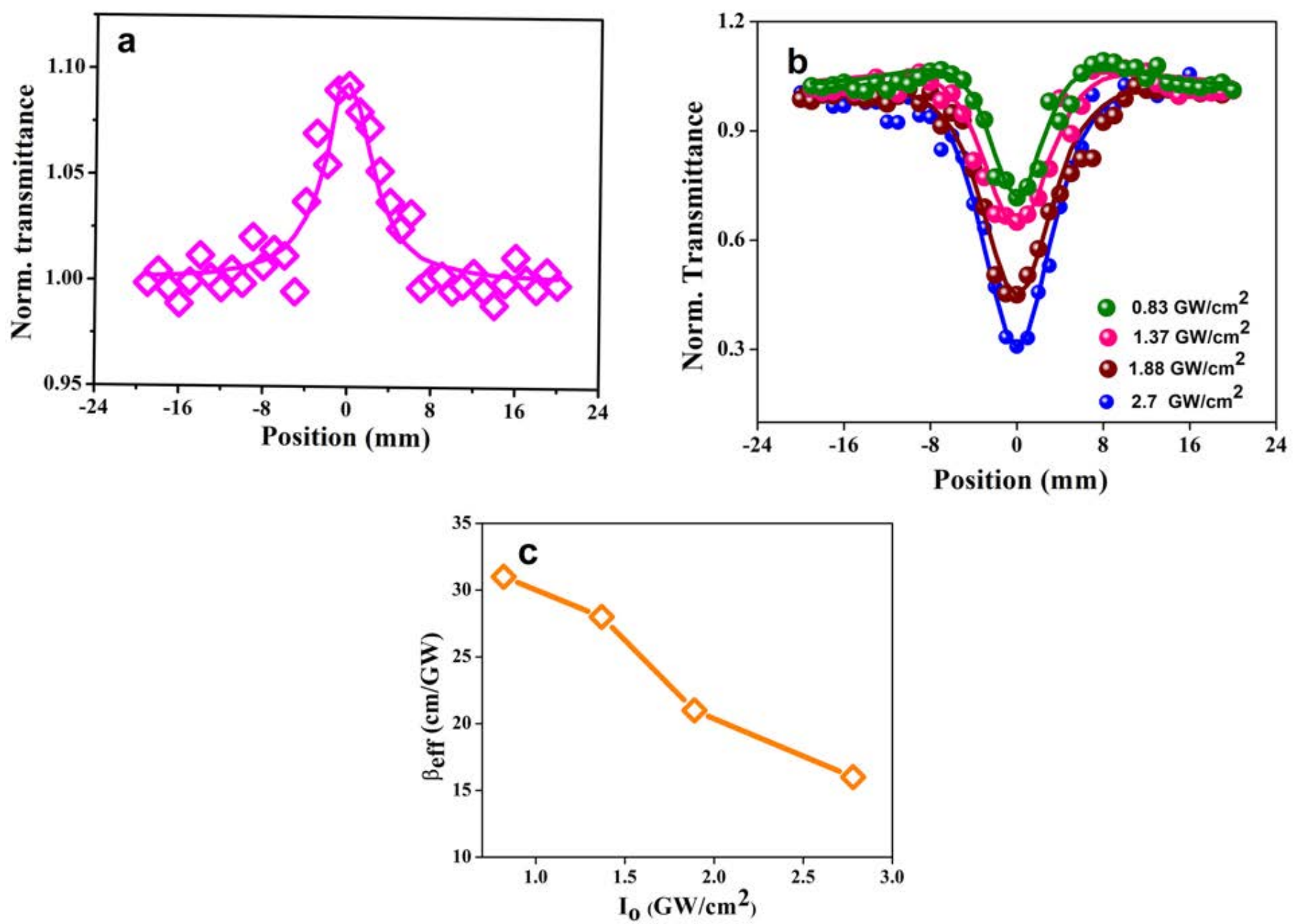

Figure 4. Open aperture (OA) Z-scan curves of Ag NPs (Linear transmittance $\sim 80 \%$ ) at an on-axis input intensity of (a) $0.27 \mathrm{GW} / \mathrm{cm}^{2}$, (b) $0.83,1.37$, and $1.88 \mathrm{GW} / \mathrm{cm}^{2}$, and (c) Nonlinear absorption coefficient as a function of on axis intensity.

Here, we employed the Z-scan technique using $7 \mathrm{~ns}$ laser pulses with $532 \mathrm{~nm}$ wavelength, at an input laser intensity of $0.27 \mathrm{GW} / \mathrm{cm}^{2}$ for the measurements. The output transmittance was found to increase when the sample moved across the focus and a gradual decrease in the transmittance was observed when it moved away from the focus. This is attributed to the absorption saturation of the conduction band electrons which occurred due to the bleaching of ground state SPR bands in the sample [19]. It was also revealed that upon further increasing the input intensity, an NLA switching behavior of the sample from SA to RSA was exhibited. In the case of RSA, the absorption cross section of the excited state was higher than that of the ground state [21]. Thus, at higher input intensities, the net absorption of the system will increase significantly due to the increased excited state transition probabilities; hence, the system behaves like an optical limiter (OL).

Metal NPs NLA properties are associated with free carriers, hot electrons, interband transition, intraband transition, etc (Figure 5) $[4,7,17,22]$. Ag NPs exhibit an intense absorption peak around $418 \mathrm{~nm}$, which originates from the $\mathrm{sp}-\mathrm{sp}$ transition and is strongly particle size dependent $[4,22,23]$. It is evident from the linear absorption spectrum obtained for Ag NPs in Figure 2a that it exhibited a definite absorption around the $532 \mathrm{~nm}$ excitation wavelength. Hence, the $532 \mathrm{~nm}$ excitation of Ag NPs is considered as nearly resonant wavelength and not fully off resonant. This leads to the equal electron population in both unoccupied and occupied states accounting for the absorption saturation displayed in Figure 5 (scheme 1). At higher input intensities, there can be two possible transitions which enable the occurrence of two photon absorption. The $\mathrm{d}$ - sp transition by excitation electrons from filled $d$ state to vacant $s p$ state is one and $s p-s p$ interband transition from 
occupied sp to unoccupied sp level is the other. The role of these two transitions in the observed NLA of Ag NPs under nanosecond pulse excitation regime is slightly lesser than the excited state absorption process (ESA) [24]. For an ESA process, the electrons are initially transferred from a ground state to an excited state at first and from there another photon is absorbed and gets transferred to higher lying states (free carrier absorption). Studies with different input intensities can further confirm the role of ESA; the determined nonlinear absorption coefficient $\beta_{\text {eff }}$ values are plotted in Figure $4 \mathrm{c}$ as the function of on axis intensity. The $\beta_{\text {eff }}$ values obtained are of the same order of magnitude reported for $\mathrm{Ag}$ NPs $[24,25]$. From the plot, it is revealed that the $\beta_{\text {eff }}$ value reduces with increasing on axis intensity and is considered as a manifestation of the ESA process. A notable reduction in the ground state population of the system and the enhanced ESA cross section due to increasing intensity can lead to an intensity dependent $\beta_{\text {eff }}$ value. If the NLA obtained only originates from genuine TPA, the $\beta_{\text {eff }}$ should remain unchanged upon increasing input intensity, due to the reason that there is an insignificant depletion of the ground state population in TPA upon increasing intensity, as it involves the virtual levels $[4,12,21]$.

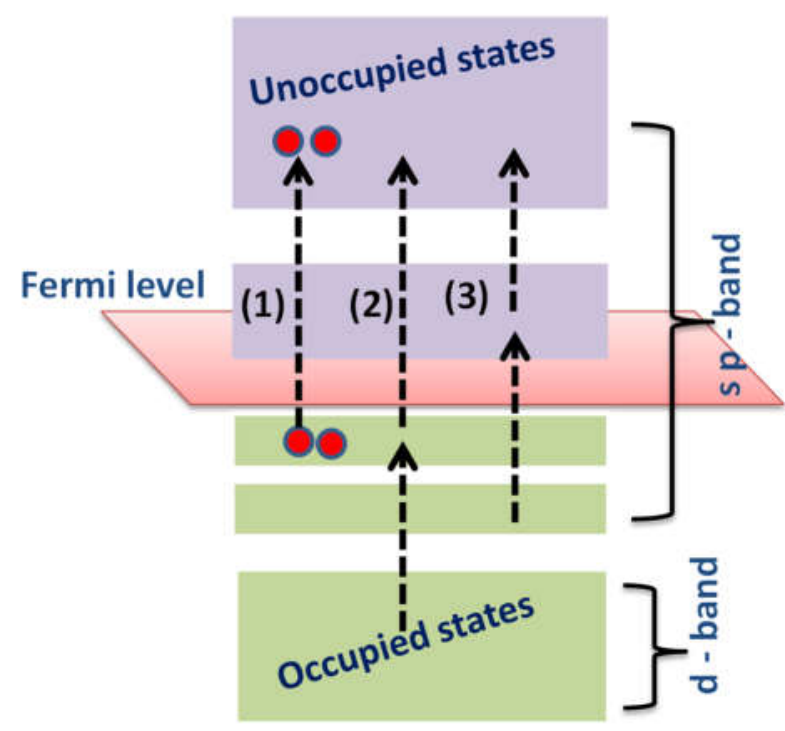

Figure 5. The scheme for excitation and successive de-excitations in Ag NPs [22]. Scheme (1) shows the SA process, in which the electron population in the lower and upper states are equal. The TPA process is represented in Schemes (2) and (3).

Optical limiters (OL) are NLO materials that exhibit uniform transmittance at lower input fluence and attenuated transmittance at elevated input fluences. Subtle optical instruments and even human eyes need to be protected from intense laser light $[4,7,14,26]$. The Ag NPs' optical limiting action was analyzed from the OA Z-scan data. The input fluence values were estimated from the various Z-positions of the open aperture Z-scan experiment. The output transmittance of the sample as a function of the input fluence is plotted in Figure 6. Figure 6a shows the input fluence against the output transmittance of Ag NPs at $0.27 \mathrm{GW} / \mathrm{cm}^{2}$. Above a threshold fluence level, an increasing transmittance is observed on increasing input fluence and is due to SA behavior of the Ag NPs. The mechanism for the SA action is discussed in the above section. Figure $6 \mathrm{~b}$ shows that with increasing input fluence, there is a decrease in transmittance (optical limiting). There are many mechanisms, such as two photon absorption, free carrier absorption, reverse saturable absorption, excited state absorption, optically induced heating, and nonlinear scattering, that are accountable for the optical limiting action of metal NPs $[7,14,26]$. The Ag NPs show an optical limiting threshold value of $5.2 \mathrm{~J} / \mathrm{cm}^{2}$, which is comparable to the values reported by many studies [26-29]. 

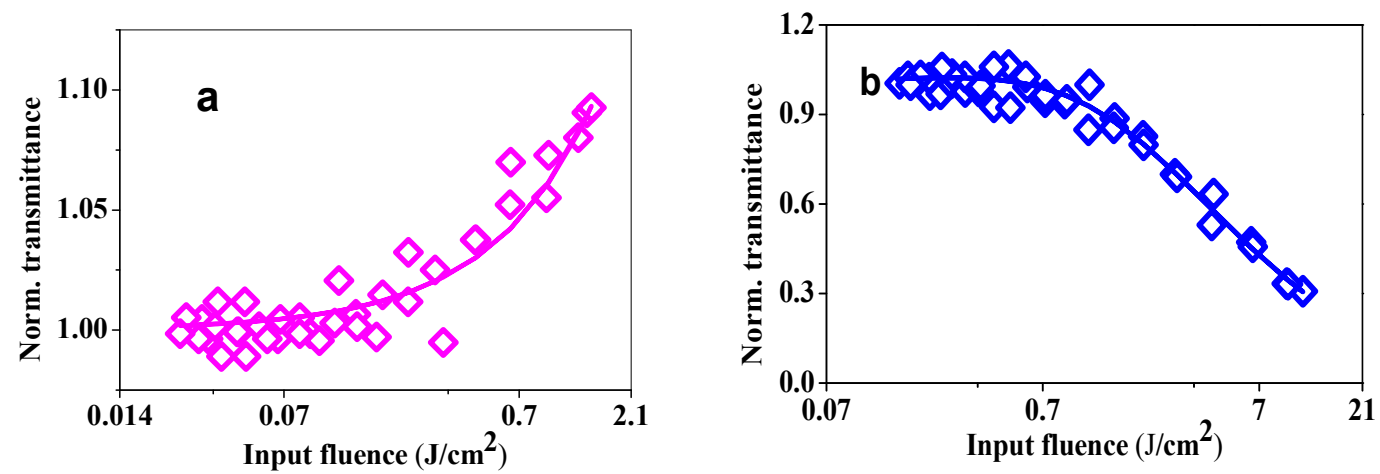

Figure 6. Input fluence versus output transmittance plot of $\mathrm{Ag} \mathrm{NPs}$ at (a) $0.27 \mathrm{GW} / \mathrm{cm}^{2}$ and (b) $2.7 \mathrm{GW} / \mathrm{cm}^{2}$.

The CA Z-scan studies help us to determine the nonlinear refraction parameters. We hired the division scheme to extract pure refractive part by removing the NLA part. This involved a direct division of the transmittance data obtained from CA Z-scan data from that obtained from the OA Z-scan data. Figure 7 shows normalized closed by open aperture Z-scan traces of Ag NPs at $0.27 \mathrm{GW} / \mathrm{cm}^{2}$ on axis peak intensity, and the symbols correspond to the obtained experimental data and the lines are numerical fit for the transmission expression [4,12,30,31] Equation (2):

$$
T=1+\frac{4 \Delta \phi_{0}\left(z / z_{0}\right)}{\left[1+\left(z / z_{0}\right)^{2}\right]\left[9+\left(z / z_{0}\right)^{2}\right]}
$$

where, $T, \Delta \phi_{0}$ indicates the normalized transmittance of the sample due to refractive nonlinearity and on-axis nonlinear phase shift at the focus, respectively. The CA Z-scan signature indicates the negative nonlinearity of Ag NPs, i.e., it exhibits a transmittance peak which is pre-focal and followed by a post focal valley. The effective index of the refraction of the beam passing through the nonlinear medium can be expressed as $n=n_{0}+n_{2} I$, where $n_{0}, n_{2}$ and $I$ are the linear refractive index, the coefficient nonlinear refraction, and the intensity of the interacting beam, respectively. Since $n_{2}$ is negative, the net refractive index should be less than $n_{0}$. Thus, $n_{2}$ causes the formation of the refractive index gradient across the beam cross section in the sample. As a result of the negative nonlinearity, the Gaussian laser beam tailors refractive index gradient with the lowest value at the beam axis and it increases as it moves towards the margin. Hence, the medium behaves like a diverging lens on incoming light, resulting in beam defocusing. The thermal variation in the index of the refraction is given by $\Delta n=(\partial n / \partial T) \Delta T$, where $(\partial n / \partial T)$ is the change in refractive index as a function of temperature and $\Delta T$ laser influenced temperature difference. These effects are predominant in cases where the interacting laser pulse width is higher than a few tens of picoseconds. The phase of the transmitting beam in the medium gets shifted by the lensing effect of the medium $[4,12,29,31]$. Th $n_{2}$ value can be calculated as per the relation $n_{2}(e s u)=\left[c n_{0} / 40 \pi\right] \gamma\left(m^{2} W^{-1}\right)$ with $\gamma=\Delta \Phi_{0} \lambda / 2 \pi I_{0} L_{e f f}$, where $n_{0}$ is the linear refractive index and $\mathrm{c}$ is the speed of the light in $\mathrm{m} / \mathrm{s}$. The $n_{2}$ value is obtained to be $1.32 \times 10^{-13} \mathrm{~cm}^{2} / \mathrm{W}$, which is larger in comparison to the reported value of $6.3 \times 10^{-14} \mathrm{~cm}^{2} / \mathrm{W}$ for Ag NPs colloids [32]; this difference can be attributed to the nanoparticle synthesis carried out using this unique laser ablation method without surfactants or chemical additions. 


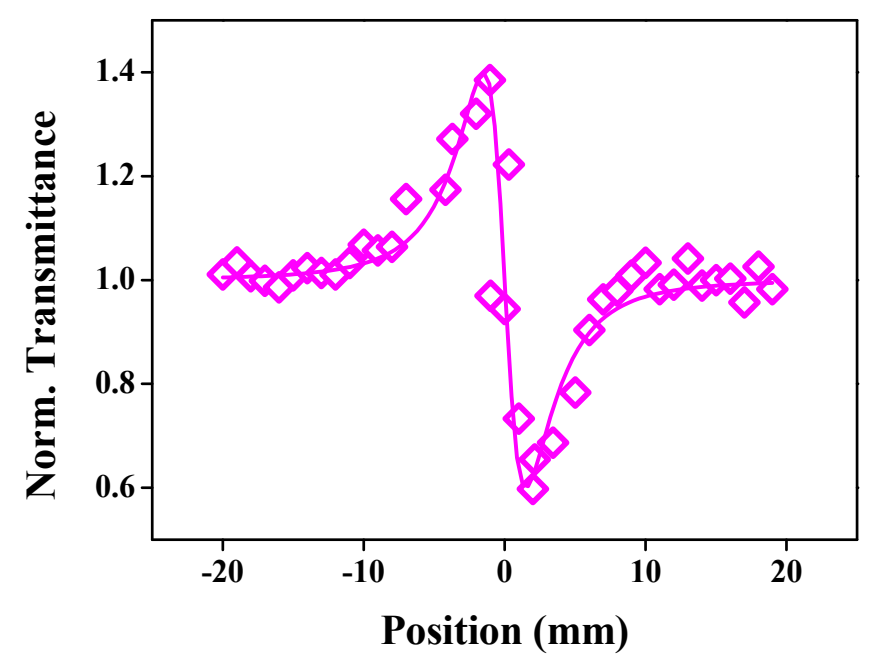

Figure 7. Pure nonlinear refraction trace of Ag NPs.

\section{Conclusions}

The pulsed laser ablation technique was employed for the synthesis of Ag nanoparticles. The absorption studies revealed that the typical SPR peak occurs around $418 \mathrm{~nm}$, and the morphology and spherical shape of the particle were confirmed from the imaging analysis. The laser excitation used for NLO studies is under ns time scales. A laser intensity sensitive switching in nonlinear absorption property (SA-RSA) was observed in the Ag NPs. The SA effect arose due to the bleaching of the conduction band electron at moderate intensity and RSA occurred due to TPA and ESA. The intensity dependent switching of NLA could be due to the interplay between different NLA mechanisms. The switching behavior of the NLA activity of Ag NPs could have widespread applications in photonics such as mode locking, optical power limiter, pulse compression, and all-optical switching.

Author Contributions: Conceptualization, E.S. and K.S.; Data curation, T.M.R.; Formal analysis, N.K.S.N., P.S. and V.S.N.; Validation, P.S., N.K.S.N. and V.S.N.; Funding acquisition, K.S.; Investigation, E.S., K.S., T.M.R. and N.K.S.N.; Project administration, E.S.; Supervision, K.S.; Writing—original draft, E.S.; Writing-review and editing, K.S. and E.S. All authors have read and agreed to the published version of the manuscript.

Funding: This research was funded by Kuwait Foundation for the Advancement of Sciences, Kuwait, grant number PR18-13SP-01.

Data Availability Statement: The data presented in this study are available on request from the corresponding author.

Acknowledgments: K.S. acknowledges Kuwait Foundation for the Advancement of Sciences (KFAS) for the research fund (PR18-13SP-01). and E.S. acknowledges SERB, India, for providing financial assistance through NPDF (File No. PDF/2020/002494).

Conflicts of Interest: The authors declare no conflict of interest.

\section{References}

1. Papagiannouli, I.; Aloukos, P.; Rioux, D.; Meunier, M.; Couris, S. Effect of the Composition on the Nonlinear Optical Response of $\mathrm{Au}^{\mathrm{x}} \mathrm{Ag}^{1-\mathrm{x}}$ Nano-Alloys. J. Phys. Chem. C 2015, 119, 6861-6872. [CrossRef]

2. Link, S.; El-Sayed, M.A. Shape and Size Dependence of Radiative, Non-radiative and Photothermal Properties of Gold Nanocrystals. Int. Rev. Phys. Chem. 2000, 19, 409-453. [CrossRef]

3. Lee, K.S.; El-Sayed, M.A. Gold and Silver Nanoparticles in Sensing and Imaging: Sensitivity of Plasmon Response to Size, Shape and Metal Composition. J. Phys. Chem. B 2006, 110, 19220-19225. [CrossRef] [PubMed]

4. Edappadikkunnummal, S.; Nherakkayyil, S.N.; Kuttippurath, V.; Chalil, D.M.; Desai, N.; Keloth, C. Surface Plasmon Assisted Enhancement in the Nonlinear Optical Properties of Phenothiazine by Gold Nanoparticle. J. Phys. Chem. C 2017, 121, 26976-26986. [CrossRef] 
5. Wang, Q.Q.; Han, J.B.; Gong, H.M. Linear and nonlinear optical properties of Ag nanowire polarizing glass. Adv. Func. Mat. 2006, 16, 2405-2408. [CrossRef]

6. Zhang, Y.X.; Wang, Y.H. Nonlinear optical properties of metal nanoparticles: A review. RSC Adv. 2017, 7, 45129-45144. [CrossRef]

7. Shijua, E.; Abhijith, T.B.; Narayana Rao, D.; Chandrasekharan, K. Phenomenal Nonlinear Optical Behavior from Gold and Silver through Au@Ag Core- Shell Nanostructures. J. Mol. Liq. 2021, 333, 115935.

8. Boltaev, G.S.; Ganeev, R.A.; Krishnendu, P.; Maurya, S.K.; Redkin, P.V.; Rao, K.S.; Zhang, K.; Guo, C. Strong third-order optical nonlinearities of Ag nanoparticles synthesized by laser ablation of bulk silver in water and air. Appl. Phys. A 2018, $124,766$. [CrossRef]

9. Pinçon, N.; Palpant, B.; Prot, D.; Charron, E.; Debrus, S. Third-order nonlinear optical response of Au:SiO 2 thin films: Influence of gold nanoparticle concentration and morphologic parameters. Eur. Phys. J. D 2002, 19, 395-402. [CrossRef]

10. Sridharan, K.; Kuriakose, T.; Philip, R.; Parkv, T.J. Transition metal (Fe, Co and Ni) oxide nanoparticles grafted graphitic carbon nitrides as efficient optical limiters and recyclable photocatalysts. Appl. Surf. Sci. 2014, 308, 19-147. [CrossRef]

11. Shiju, E.; Bharat, M.; Narendran, N.K.S.; Rao, D.N.; Chandrasekharan, K. Optical diode activity in an axially asymmetric nonlinear medium incorporated with phenothiazine and silver nanoparticles. Opt. Mater. 2020, 99, 109557-109562. [CrossRef]

12. Shiju, E.; Narendran, N.K.S.; Rao, D.N.; Chandrasekharan, K. A phenothiazine-silver hybrid system exhibiting switching and photo-induced enhancement in nonlinear optical absorption. New J. Chem. 2019, 43, 7962-7971.

13. Unnikrishnan, K.P.; Nampoori, V.P.N.; Ramakrishnan, V.; Umadevi, M.; Vallabhan, C.P.G. Nonlinear optical absorption in silver nanosol. J. Phys. D. Appl. Phys. 2003, 36, 1242-124513. [CrossRef]

14. Remya, T.M.; Shiju, E.; Shandev, P.P.; Chandrasekharan, K.; Suja Haridas Unnikrishnanan, P.A. Enhanced Nonlinear Absorption and Efficient Optical Limiting Action of a Few 1,3,4-Oxadiazole Based Donor-Acceptor Systems. J. Mat. Sci. 2021, 56, 3035-3048. [CrossRef]

15. Shiju, E.; Siji Narendran, N.K.; Narayana Rao, D.; Chandrasekharan, K. Enhanced nonlinear absorption and efficient power limiting action of Au/Ag@ graphite core-shell nanostructure synthesized by laser ablation. Nano Express 2020, 1, 030026.

16. Semaltianos, N. Nanoparticles by Laser Ablation. Crit. Rev. Solid State Mater. Sci. 2010, 35, 105-124. [CrossRef]

17. Sreeramulu, V.; Haldar, K.K.; Patra, A.; Rao, D.N. Nonlinear Optical Switching and Enhanced Nonlinear Optical Response of Au-CdSe Heteronanostructures. J. Phys. Chem. C 2014, 118, 303333. [CrossRef]

18. Jayabalan, J.; Singh AChari, R.; Oak, S.M. Ultrafast third-order nonlinearity of silver nanospheres and nanodiscs. Nanotechnology 2007, 1831, 315704. [CrossRef]

19. Hari, M.; Mathew, S.; Nithyaja, B.; Joseph, S.A.; Nampoori VP, N.; Radhakrishnan, P. Saturable and reverse saturable absorption in aqueous silver nanoparticles at off-resonantwavelength. Opt. Quantum Electron. 2012, 43, 49-58. [CrossRef]

20. Ajami, A.; Husinsky, W.; Svecova, B.; Vytykacova, S.; Nekvindova, P. Saturable absorption of silver nanoparticles in glass for femtosecond laser pulses at $400 \mathrm{~nm}$. J. Non-Cryst. Solids 2015, 426, 159-163. [CrossRef]

21. Edappadikkunnummal, S.; Prasannan, D.; Francis, J.; Desai, N.R.; Keloth, C. An insight into phenomenal optical non-linearities arising from synergistic relationship between selected BODIPYs and noble metal nanoparticles. Appl. Organomet. Chem. 2021, 35, e6029. [CrossRef]

22. Philip, R.; Chantharasupawong, P.; Qian, H.; Jin, R.; Thomas, J. Evolution of nonlinear optical properties: From gold atomic clusters to plasmonic nanocrystals. Nano Lett. 2012, 12, 4661-4667. [CrossRef]

23. Aikens, C.; Li, M.; Schatz, G.C. From discrete electronic states to plasmons: TDDFT optical absorption properties of Agn ( $\mathrm{n}=10$, 20, 35, 56, 84, 120) tetrahedral clusters. J. Phys. Chem. C 2008, 112, 11272-11279. [CrossRef]

24. Parvathy, N.; Jiya, J.; Nithin, J.; Sivakumaran, V.; Reji, P.; Rodolphe, A.; Sabu, T.; Nandakumar, K. Fabrication of Silver-Decorated Graphene Oxide Nanohybrids via Pulsed Laser Ablation with Excellent Antimicrobial and Optical Limiting Performance. Nanomaterials 2021, 11, 880.

25. Jijuan, J.; Jun, W.; Tong, W.; Yachen, G. Nonlinear Absorption and Ultrafast Dynamics of Ag Nanoparticle. Photonics 2021, 8, 233.

26. Anand, B.; Addo, S.; Muthukumar Sai, V.; Siva Sankara Sai, S.; Philip, R.; Mitra, S. Improved Optical Limiting in Dispersible Carbon Nanotubes and Their Metal Oxide Hybrids. Carbon 2011, 49, 4767-4773. [CrossRef]

27. Sudheesh, P.; Siji Narendran, N.K.; Chandrasekharan, K. Third-Order Nonlinear Optical Responses in Derivatives of Phenylhydrazone by Z-Scan and Optical Limiting Studies-Influence of Noble Metal Nanoparticles. Opt. Mater. 2013, 36, 304-309. [CrossRef]

28. Udayabhaskar, R.; Karthikeyan, B.; Sreekanth, P.; Philip, R. Enhanced Multi-Phonon Raman Scattering and Nonlinear Optical Power Limiting in ZnO:Au Nanostructures. RSC Adv. 2015, 5, 13590-13597. [CrossRef]

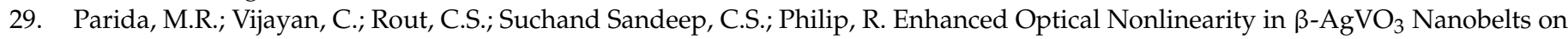
Decoration with Ag Nanoparticles. Appl. Phys. Lett. 2012, 100, 1-6. [CrossRef]

30. Jayakrishnan, K.; Joseph, K.A.; Bhattathiripad, J.; Ramesan, M.T.; Siji Narendran, N.K.; Chandrasekharan, K. Reverse Saturable Absorption Studies in Polymerized Indole-Effect of Polymerization in the Phenomenal Enhancement of Third Order Optical Nonlinearity. Opt. Mater. 2016, 54, 252-261. [CrossRef] 
31. Kuladeep, R.; Jyothi, L.; Prakash, P.; Mayank Shekhar, S.; Durga Prasad, M.; Narayana Rao, D. Investigation of optical limiting properties of Aluminium nanoparticles prepared by pulsed laser ablation in different carrier media. J. Appl. Phys. 2013, 114, 243101. [CrossRef]

32. Corrêa, N.F.; Santos, C.E.; Valadão, D.R.; de Oliveira, L.F.; Dupont, J.; Alencar, M.A.; Hickmann, J.M. Third-order nonlinear optical responses of colloidal Ag nanoparticles dispersed in BMI.BF4 ionic liquid. Opt. Mater. Expr. 2016, 6, 1. [CrossRef] 